\title{
GENERAL OVERVIEW OF GAN DEVICES AND TRANSPORT PROPERTIES OF ALGAN/GAN HEMT STRUCTURES - IMPACT OF DISLOCATION DENSITY AND IMPROVED DESIGN
}

\author{
${ }^{1}$ Eduard HULICIUS, ${ }^{1,2}$ František HÁJEK, ${ }^{1}$ Alice HOSPODKOVÁ, ${ }^{1}$ Pavel HUBÍK,

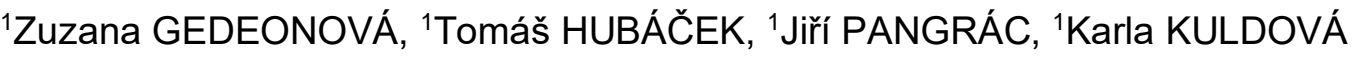 \\ 1'Institute of Physics CAS, v.v.i., Prague, Czech Republic, EU, hulicius@fzu.cz \\ ${ }^{2}$ Faculty of Nuclear Sciences and Physical Engineering, CTU in Prague, Czech Republic, EU
}

https://doi.org/10.37904/nanocon.2021.4309

\begin{abstract}
GaN-based nanostructures are used for many present semiconductor devices. The main topics are structures for blue LEDs and LDs, but there are also other interesting and important GaN devices namely for power electronics, scintillators and detectors as well as High Electron Mobility Transistors (HEMT). Several of our previous NANOCON contributions were devoted to the scintillator and detector structures; one was a description of optimization of HEMT structures and their growth parameters.
\end{abstract}

Keywords: GaN devices, HEMT, MOVPE epitaxy, dislocation

\section{INTRODUCTION}

This contribution is devoted to a general review of GaN power electronic devices, a comparison with other materials (mainly $\mathrm{Si}$ and $\mathrm{SiC}$ ), a description of some fields of application and a future forecast.

The second part will describe our recent results - the influence of dislocation density on transport properties of HEMT structures is reported. Experimental results, obtained on HEMT structures prepared in the same growth run on templates with different dislocation densities, are compared. We show experimentally that lowering the dislocation density considerably increases the electron mobility in 2DEG. It is shown that the dislocation density has also a significant influence on the Fermi level position in GaN. Presented results suggest that reduction of the dislocation density can improve the performance of high frequency GaN HEMTs applications.

High frequency power electronic devices have many application fields, as it is shown in Figures $\mathbf{1 a}$ and $\mathbf{b}$.

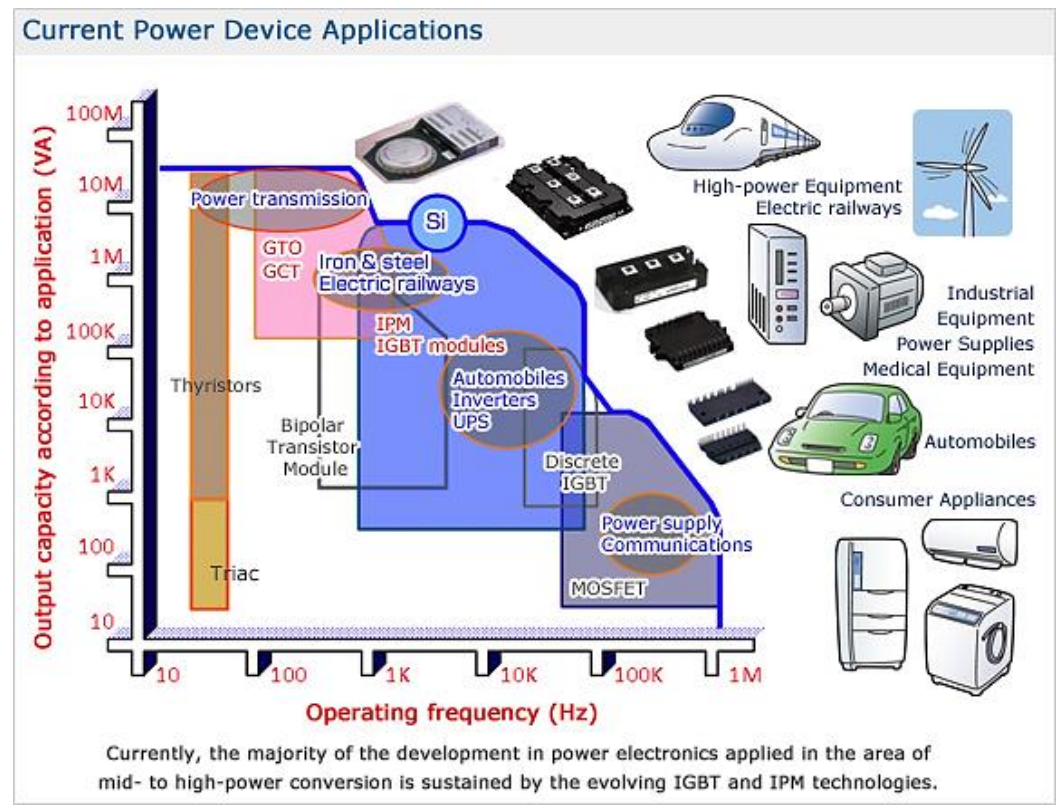

a) Output power $(W)$ versus operating frequencies $(\mathrm{Hz})$ for some current and future devices. The majority of the development in power electronic applied in the area of mid- to high-power conversion is sustained by the evolving IGBT and IPM technologies. 


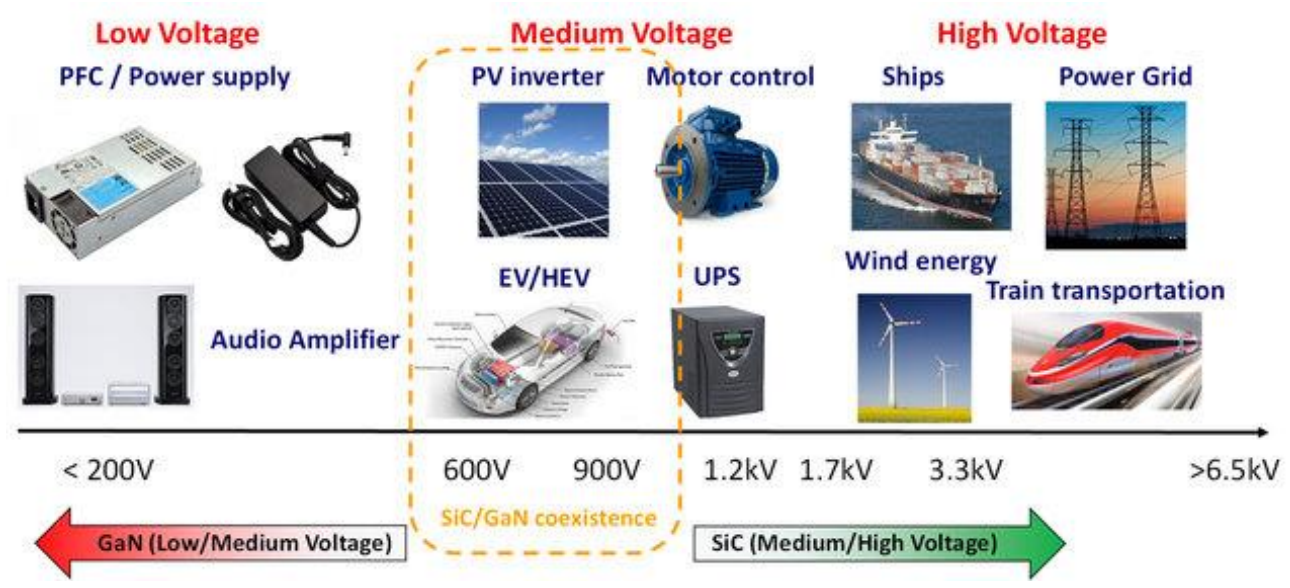

b) Different devices (current and future) according to the maximum applied "Voltages".

Figure 1a, b Application fields for three the most important semiconductor materials from the output power versus operating frequencies and voltage point of view.

There are three most important and promising materials: $\mathrm{Si}, \mathrm{SiC}$ and $\mathrm{GaN}$. Devices are based on different structures - see Figure 2, but the material properties are determinative (Figure $\mathbf{2 b}$ ). Of course, there exist many other interesting materials, as $\mathrm{Ga}_{2} \mathrm{O}_{3}$, $\mathrm{GaAs}$, InP or old school vacuum tubes - see Figure $\mathbf{2 a}$, at the edge of electric device parameters. However, the main industrial players are only three. They also meet the requirements on temperature and radiation resistance as well as sufficient lifetime.

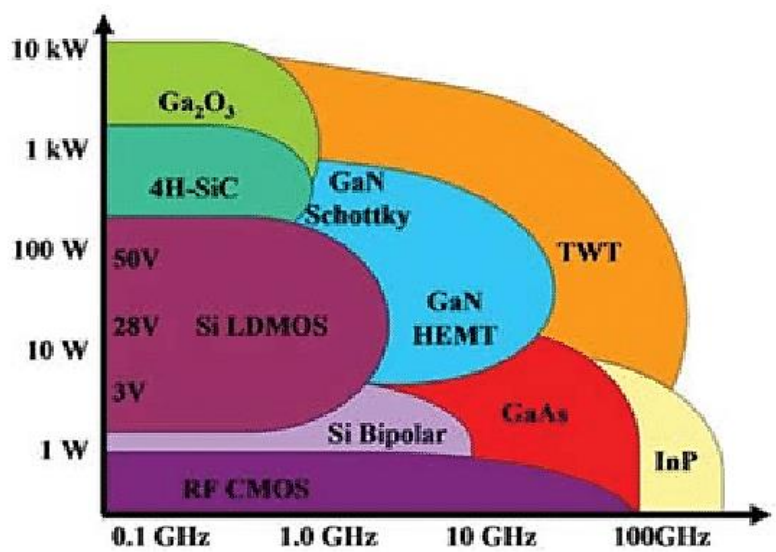

(a)

Figure 2 Comparison of some semiconductor materials and structures for different Output power versus Operating frequencies - upper part (a), and key physical parameters, which are responsible for device and structure limits - lower part (b). (TWT is

Traveling-wave tube amplifier, a specialized vacuum tube that is used in electronics to amplify radio frequency (RF) signals in the microwave range.)

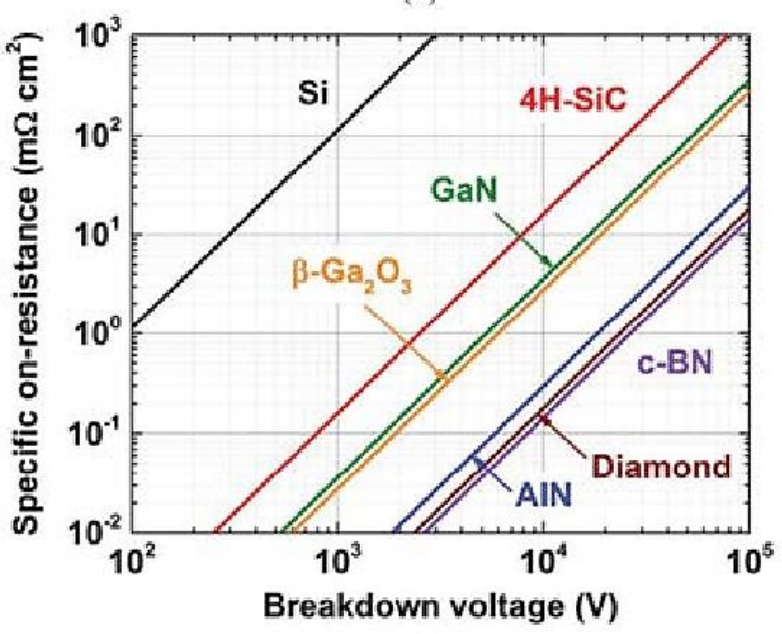

(b)

We prepare and study InGaN/GaN and AIGaN/GaN scintillator and HEMT structures not only for scientific research, but also for industrial applications for the last 8 years, thus we will put here stress on GaN. In Figure 3, there are shown development and predictions of GaN power devices market. 


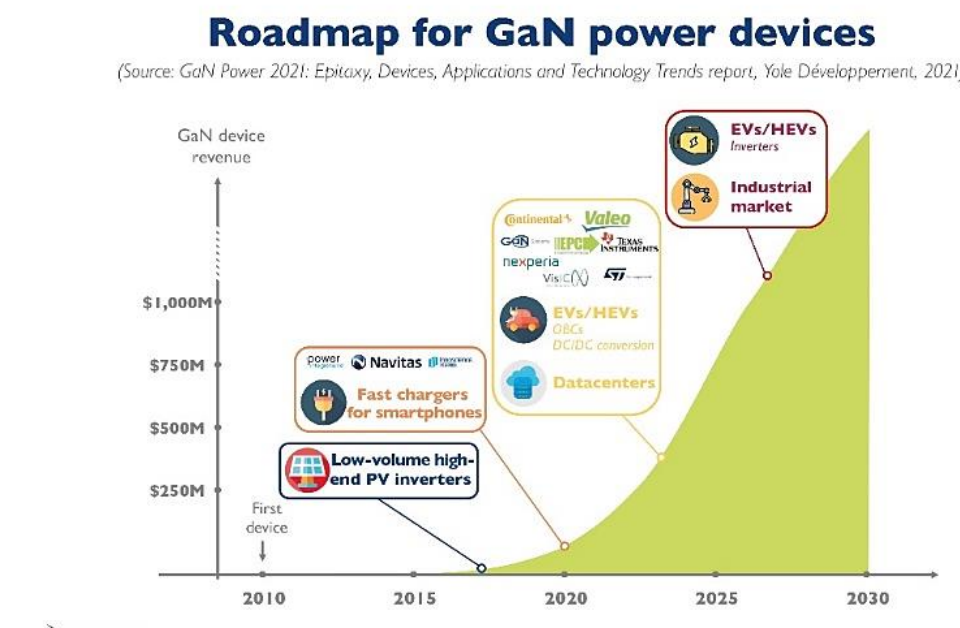

$Y^{\text {Deveropopenent }}$
a) Rather optimistic YOLE prediction (2021), starting from the "Low-volume high-end PV inverters (2017)" over the "Fast chargers for smartphones (2020)", "E-cars, and Datacenters (2023)" to the "EVs/HVs Inverters and Industrial market (2027)".

GaN for power electronics application: evolution of the market with two scenarios (Source: Power GaN 2018: Epitaxy, Devices, Applications and Technology Trends,

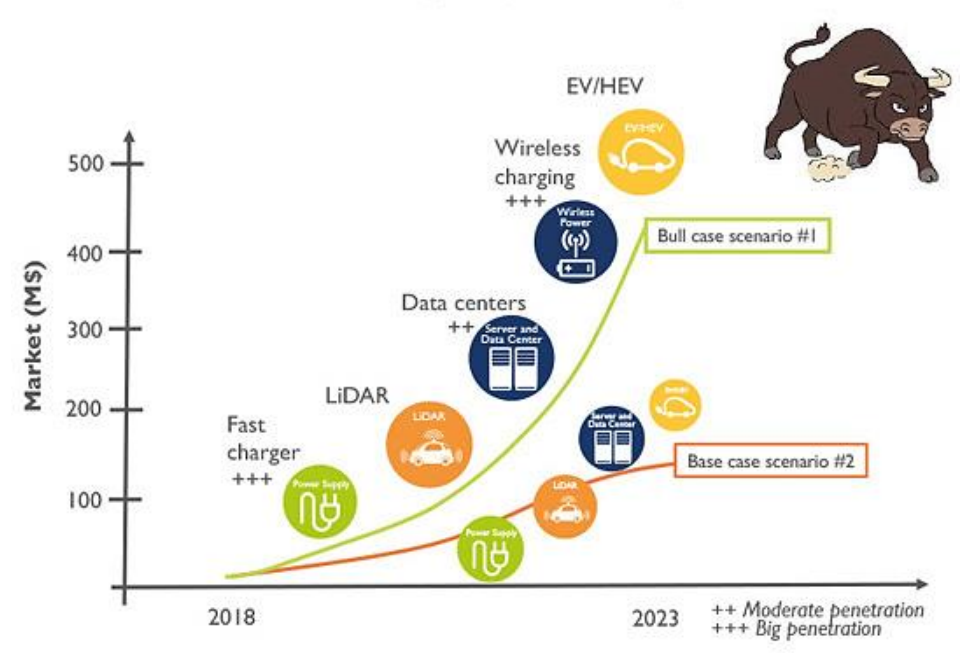

b) GaN power devices market forecasts. Bull case scenario = higher or moderate penetration and earlier realizations and as new: "Wireless charging (2022)" It is about $1 / 4$ of all GaN possible applications.

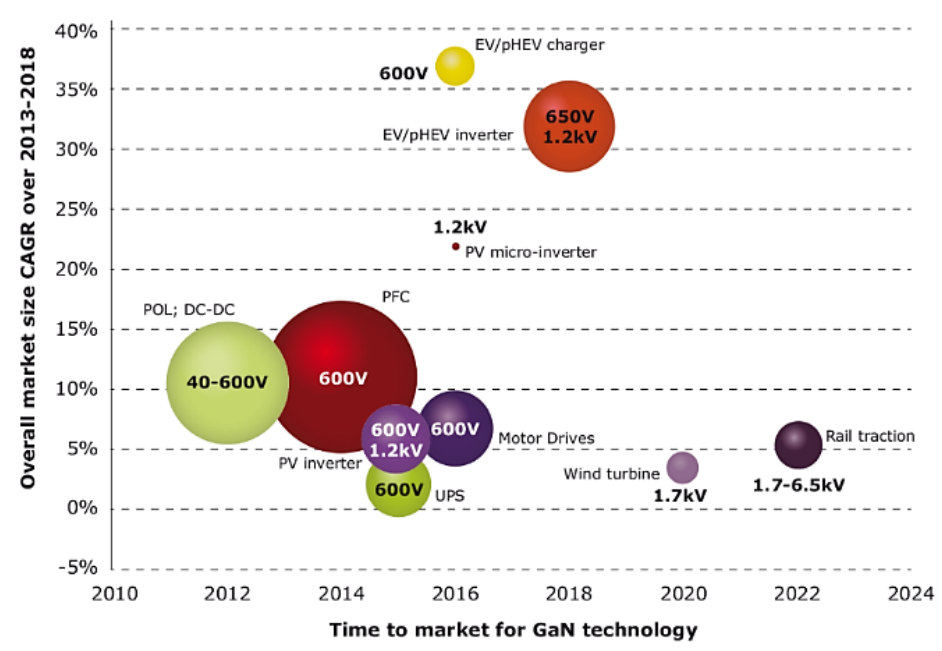

c) GaN devices development versus voltage. Dark red 600 V Power Factor Correction (PFC) single-phase AC/DC; Green 40-600 V DC/DC converters and UPS; Red 650-1200 V-EV/pHEV inverter; Violet 600-1200 V-PV inverter, Motor drivers and Rail traction.

Figure 3a, b, c GaN power devices market forecast for different application fields and producer companies 
Global Smart Lighting Market Size (2018-2023)

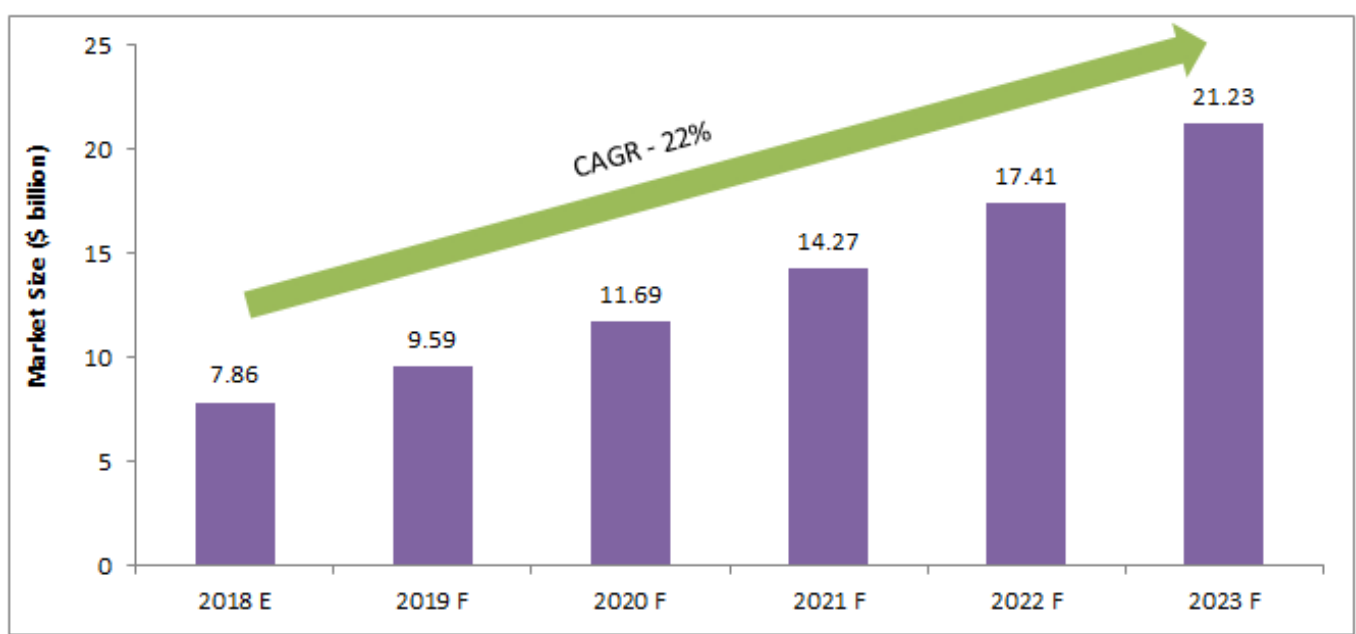

Source: Beroe Analysis

Figure $4 \mathrm{GaN}$ LED market predictions are more than order of magnitude higher than GaN power devices

In comparison with GaN blue/white LED huge market - see Figure 4, the GaN power devices market is only $10 \%$ of the LED market, but absolute value of the GaN power devices market is currently about 2 billion USD with estimated strong growth, as it is shown in Figure 3.

\section{RESULTS OF OUR RESEARCH ON ALGAN/GAN HEMT STRUCTURE}

\subsection{Regular AIGaN/GaN HEMT structure (without AIGaN back barrier)}

In our laboratory were prepared different HEMT structures [1]. The scheme are depicted in Figure 5. Structures were prepared on three types of $\mathrm{GaN}$ templates differing in dislocation density: GaN substrate with a very low dislocation density of approximately $10^{6} \mathrm{~cm}^{-2}$, and two templates on sapphire differing by the GaN buffer technology leading to lower (LDD) and higher dislocation density (HDD). For comparison of transport properties of these structures, see Table 1.

a)

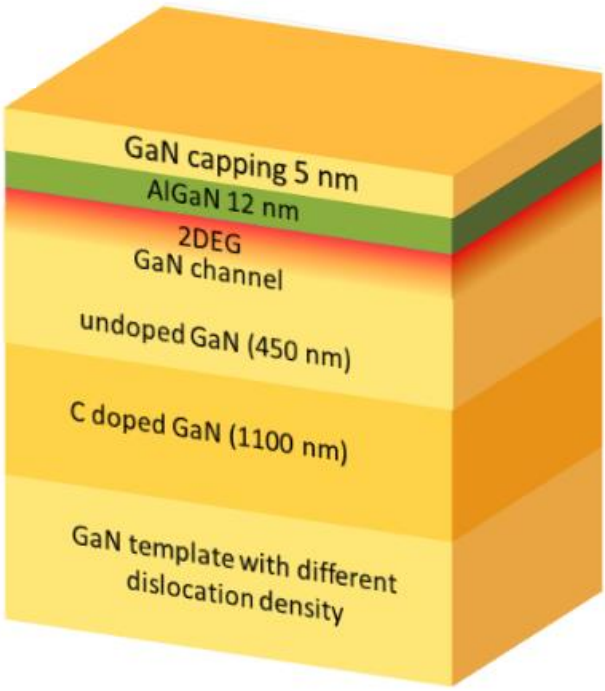

b)

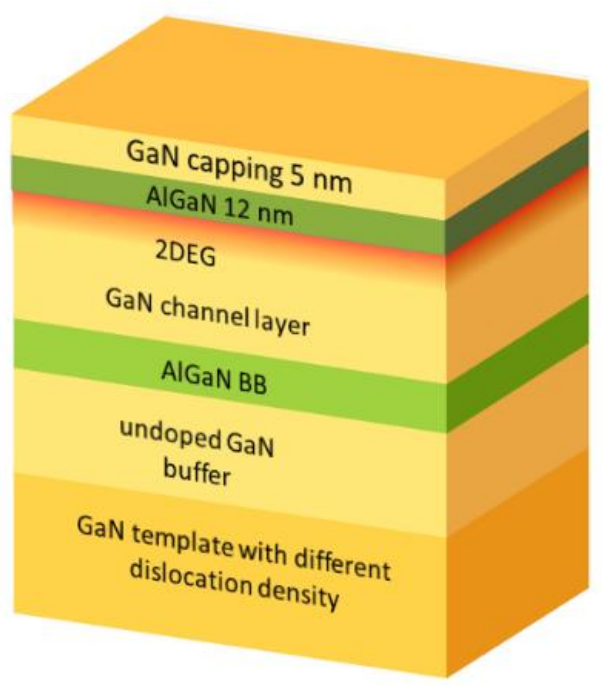

Figure 5 Scheme of (a) conventional HEMT structure and (b) HEMT structure with AIGaN back-barrier 
Table 1 Dislocation density according to XRD, concentration and mobility of samples on different templates

\begin{tabular}{|c|c|c|c|c|c|}
\hline Sample & $\begin{array}{c}\text { Type of template and } \\
\text { substrate }\end{array}$ & $\begin{array}{c}\text { Dislocation } \\
\text { densities }\left(\mathrm{cm}^{-2}\right)\end{array}$ & $\begin{array}{c}\text { Apparent 2DEG } \\
\text { concentration } \\
\text { from } \mathbf{C V}\left(\mathrm{cm}^{-2}\right)\end{array}$ & $\begin{array}{c}\text { Sheet Hall } \\
\text { concentration } \\
\left(\mathrm{cm}^{2}\right)\end{array}$ & $\begin{array}{c}\text { Mobility } \\
\left(\mathrm{cm}^{2} / \mathrm{Vs}\right)\end{array}$ \\
\hline HDD template & HDD on sapphire & $2.79 \times 10^{10}$ & $1.20 \times 10^{13}$ & $8.77 \times 10^{12}$ & 1187 \\
\hline LDD template & LDD on sapphire & $9.36 \times 10^{8}$ & $1.18 \times 10^{13}$ & $1.56 \times 10^{13}$ & 1360 \\
\hline PLDD & LDD on patterned sapphire & $6.81 \times 10^{8}$ & $1.17 \times 10^{13}$ & $1.09 \times 10^{14}$ & 539 \\
\hline GaN substrate & GaN substrate & $3 \times 10^{6}$ & $9.78 \times 10^{12}$ & $3.18 \times 10^{15}$ & 798 \\
\hline
\end{tabular}

Surprisingly, poor mobility results were obtained on samples prepared on patterned sapphire or GaN substrates with lower dislocation densities. According to Hall measurements, these samples also revealed very high sheet carrier concentrations. Capacity measurements giving carrier profiles revealed that this unexpectedly high carrier concentration is not caused by carriers in the 2DEG channel, but by carriers from a deeper layer in the heterostructure. In structures prepared on LDD substrate, deeper layers have low resistivity, and conductive channels parallel to 2DEG are dominant in Hall measurement. Parallel currents in nitride structures grown on sapphire substrates were also previously reported [2]. These parasitic channels not only influence Hall measurement results, but they are also detrimental for HEMT applications. In the case of HDD structures, undoped $\mathrm{GaN}$ and $\mathrm{C}$-doped $\mathrm{GaN}$ regions become highly resistive and they insulate the channel region from the deeper layer of the structure. Thus, only for the HDD structures was the 2DEG active region measured by Hall measurement. For the same reason, it is not a problem to grow industrial HEMT structures on Si substrates, where a high density of dislocations is formed.

In order to study the influence of dislocation density on transport properties of HEMT structures a method for improved localization of electrons in the channel region is required. Such a solution could also be useful for the application of HEMT structures with a low dislocation density. We have found that the widely used carbon doping of buffer layers (see conventional HEMT structure in Figure 5a is not reliable and does not provide sufficient isolation, especially for structures with a much lower dislocation density. The lower resistance of Cdoped layers causes this, when the dislocation density is low. Fe doping creating a semi-insulating buffer may help to solve this problem [3]. AIN/GaN superlattice can also be used [4]. Another possible solution, which has been recently published [5,6], and which we have decided to follow and investigate in more detail, is the introduction of a so called "back barrier" (BB) layer below the channel region, see Figure $\mathbf{5 b}$.

\subsection{HEMT structures with AIGaN back barrier - experimental results}
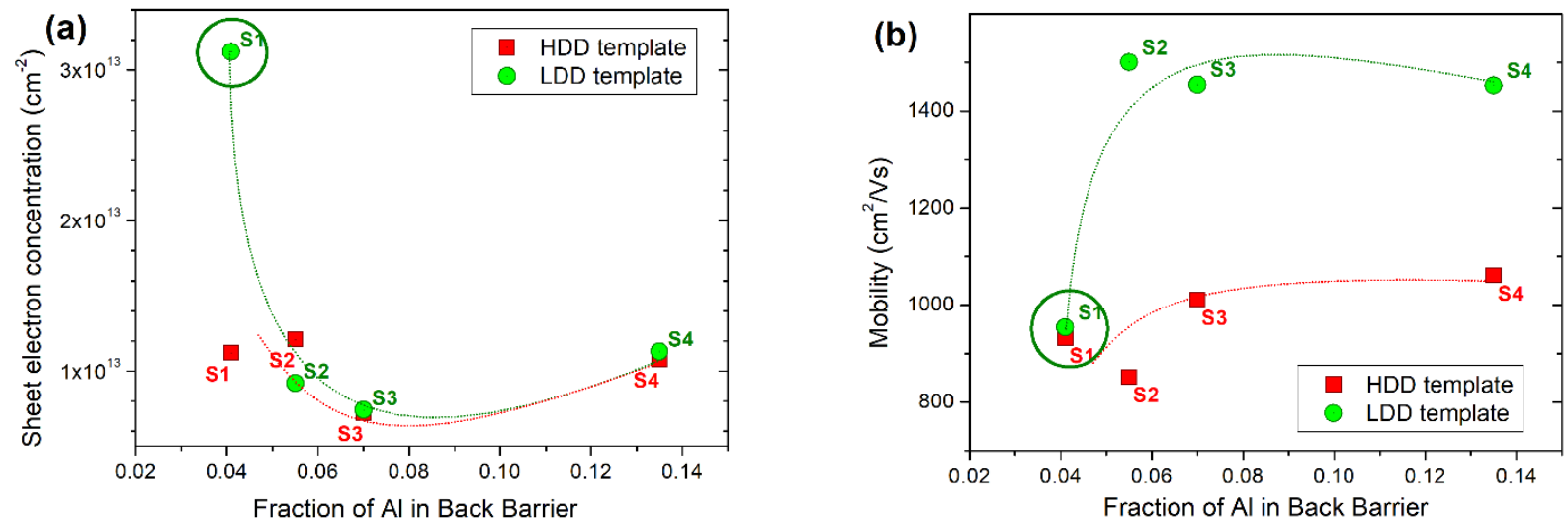

Figure 6 (a) Measured sheet carrier concentration and (b) mobility as a function of Al concentration in AIGaN BB for HDD (red squares) and LDD (green circles) samples. The results marked by circle suggest leaking AIGaN BB for LDD sample S1, [1]. 
Three sets of structures were prepared to identify optimal parameters for a structure with an AIGaN BB and to compare experimental and simulated results. Particular sets of structures used to study the influence of BB composition, thickness of $\mathrm{BB}$ and $\mathrm{GaN}$ channel layer thickness are color-coded in the table. All structures were prepared on LDD and HDD templates during the same procedure to study the influence of dislocation density on transport properties in 2DEG.

Results of transport measurements are shown in Figure 6. The most obvious result is that, in the case of structures prepared on HDD templates, structure parameters do not significantly influence electron mobility in the 2DEG channel.

\section{CONCLUSION}

We have shown that reduction of dislocation density considerably increases electron mobility in 2DEG. All presented results support our expectation that a suitably designed AIGaN back barrier can help to prevent this phenomenon. The best results were obtained for an $\mathrm{Al}_{x} \mathrm{Ga}_{1-x} \mathrm{~N} \mathrm{BB}$ composition, with $\mathrm{x}$ in the range 0.07-0.15, a BB thickness of $17 \mathrm{~nm}$ and a GaN channel layer thickness $>140 \mathrm{~nm}$. For structures prepared on HDD buffer, parallel currents through deeper parts of the heterostructure were not detected, even in the case where no BB or other isolation layer was used. We did not observe any influence of BB parameters on transport properties of samples prepared on HDD templates.

The exact mechanism of how the dislocation density significantly influences 2DEG transport will be the subject of further investigation. Since scattering on dislocations was excluded as a significant scattering mechanism in 2DEG, other mechanisms, such as scattering on ionized impurities surrounding dislocations or the influence of the interface morphology, may be responsible for lowering the electron mobility in structures with a high dislocation density. Finally, the presented results suggest that reduction of dislocation density increases electron mobility in 2DEG, which is beneficial for GaN HEMTs high frequency applications.

\section{ACKNOWLEDGEMENTS}

The authors acknowledge support from MEYS project LTAIN19163; from CzechNanoLab project LM2018110 at LNSM Res. Infrastructure and from the Operational Programme RDE financed by EU Structural and Invest. Funds and by MEYS (Project No. SOLID21 CZ.02.1.01/0.0/0.0/16_019/0000760).

\section{REFERENCES}

[1] HAJEK, F. et al. Transport properties of AIGaN/GaN HEMT structures with back barrier: impact of dislocation density and improved design. Sem. Sci. and Technol. 2021, vol. 36, p. 075016.

[2] LOOK, D. C., MOLNAR, R. J. Degenerate layer at GaN/sapphire interface. Appl. Phys. Lett. 1997, vol. 70, pp. 3377-3379.

[3] BOUGRIOUA, Z, et al. Some benefits of Fe doped less dislocated GaN templates for AIGaN/GaN HEMTs grown by MOVPE. Phys. Status Solidi A. 2005, vol. 202, pp. 536-544.

[4] SHEN, X. Q., et al. High-quality GaN film and AIGaN/GaN HEMT grown on 4-inch Si (110) substrates by MOCVD using an ultra-thin AIN/GaN superlattice interlayer. Phys. Status Solidi B. 2015, vol. 252, pp. 10751078.

[5] GU, Y., CHANG, D., SUN, H., ZHAO, J., YANG, G., DAI, Z., DING, Y. Theoretical Study of InAIN/GaN High Electron Mobility Transistor (HEMT) with a Polarization-Graded AIGaN Back-Barrier Layer. Electronics. 2019, vol. 8, p. 885 .

[6] LIU, X., et al. Analysis of the back-barrier effect in AIGaN/GaN high electron mobility transistor on free-standing GaN substrates. J. Alloys Compd. 2020, vol. 814, p. 152293. 